\title{
ON PERTURBATION OF CONTINUOUS MAPS
}

\author{
MARIA C. CARBINATTO \\ ICMC, USP, Caixa Postal 668 \\ 13560-970 São Carlos, SP, Brazil \\ E-mail: mdccarbi@icmc.sc.usp.br
}

\begin{abstract}
In [1], the concept of singular isolating neighborhoods for a continuous family of continuous maps was presented. The work was based on Conley's result for a continuous family of continuous flows (cf. [2]). In this note, we study a particular family of continuous maps to illustrate the results in [1].
\end{abstract}

1. Introduction. The Conley index theory has become an important tool for qualitative studies of dynamical systems. Two main ingredients of the theory are the existence of isolating neighborhoods and index pairs. In [2], Conley presented how to circumvent the problem of losing the isolation property in the context of perturbations of a family of continuous flows (cf. [2, Lemma B] and [2, Theorem C]). In his setup, a family of continuous flows did not have the isolation property for a particular flow and isolating neighborhoods could be found for nearby flows. Conley defined the concept of singular isolating neighborhoods and gave conditions to determine them. In [5], Mischaikow, Mrozek and Reineck proved the existence of singular index pairs also for a family of continuous flows (cf. [5, Theorem 1.15]). The discrete case was considered in [1] where the discrete version of Conley's theorem was given. The problem of constructing singular index pairs for continuous maps has been treated as well. The goal of this note is to present a perturbation problem that illustrates the results proved in [1].

We will use the logistic map to define a family of continuous maps. We start with the following family of $C^{1}$ functions from $\mathbb{R}^{2}$ to $\mathbb{R}$. Let $\varepsilon \geq 0, f, g^{\varepsilon}, h: \mathbb{R}^{2} \rightarrow \mathbb{R}$ be $C^{1}$ functions given by

$$
\begin{aligned}
f(x, y) & =x(3.3-y)(1-x) \\
g^{\varepsilon}(x, y) & =y+\varepsilon y h(x, y) .
\end{aligned}
$$

We will consider the following family of continuous maps from $[0,1] \times[0,3.3]$ to $\mathbb{R}^{2}$ :

$$
F^{\varepsilon}(x, y)=\left(f(x, y), g^{\varepsilon}(x, y)\right) .
$$

1991 Mathematics Subject Classification: Primary 58F30; Secondary 54H20.

The paper is in final form and no version of it will be published elsewhere. 
Some conditions will be imposed on the function $h$. However, we postpone the description of them to Section 3. When $\varepsilon=0$, we will have the map $f_{0}(x, y):=(f(x, y), y)$. Recall that given a set $N \subset \mathbb{R}^{2}$, its maximal invariant set under $f_{0}$ is defined by

$$
\operatorname{Inv}\left(N, f_{0}\right):=\left\{x \in N \mid \exists\left\{x_{n}\right\}_{n=-\infty}^{\infty} \subset N \text { such that } x_{n+1}=f_{0}\left(x_{n}\right) \text { and } x_{0}=x\right\},
$$

a compact set $N$ is an isolating neighborhood if

$$
S:=\operatorname{Inv}\left(N, f_{0}\right) \subset \operatorname{Int}(N),
$$

where $\operatorname{Int}(N)$ denotes the interior of the set $N$, and $S$ is called isolated invariant set.

The continuation property under perturbation of the Conley index implies that if $N$ is an isolating neighborhood for $f_{0}$, then there exists $\bar{\varepsilon}>0$ such that for all $\varepsilon \in[0, \bar{\varepsilon}], N$ is an isolating neighborhood for $F^{\varepsilon}$. However, in Section 3, we will introduce a compact set which it is not an isolating neighborhood for $f_{0}$ and it does have the isolation property for $\varepsilon>0$. Compact sets with this property are called singular isolating neighborhoods. In Section 2, we present the precise definition of singular isolating neighborhoods and state the main result of [1] that presents sufficient conditions to determine singular isolating neighborhoods. In Section 3, we return to the family of continuous maps $F^{\varepsilon}$.

Notice that for $\varepsilon=0$, the map $f_{0}$ has the logistic map in its first coordinate which presents a well known dynamics. Restricting the variation of $y$ to the interval $[0,3.3]$, the function $x \mapsto x(3.3-y)(1-x)$ presents fixed points and 2-periodic points. In Section 3 , we recall the computations of these points. Working with a particular family of $C^{1}$ functions, $h_{\lambda}$ (its definition will be given in Section 4), an index computation for $F^{\varepsilon}$ (in fact, $F_{\lambda}^{\varepsilon}$ ) is done. In Section 4 , we present this computation and show that it yields some information about the dynamics of $F_{\lambda}^{\varepsilon}$.

2. A perturbation theorem. This section is devoted to the main notation and results for the perturbation problem. For further details on the basic definitions, we refer the reader to [1], [2] and [7]. For proofs of the results described in this section, see [1].

In what follows, $\mathbb{Z}$ denotes the set of integer numbers, $\mathbb{Z}^{+}=\{i \in \mathbb{Z}: i \geq 0\}$ and $\mathbb{Z}^{-}=\{i \in \mathbb{Z}: i \leq 0\}$. Let $X$ denote a locally compact metric space and $f: X \rightarrow X$ be a continuous map. Given $x \in X$, a full left solution through $x$ is a continuous map $\sigma: \mathbb{Z}^{-} \rightarrow X$ such that

(a) $\sigma(0)=x$,

(b) $f(\sigma(i-1))=\sigma(i)$ for all $i \in \mathbb{Z}^{-}$.

We can extend a left solution through $x$ to a function defined on $\mathbb{Z}$ by setting $\sigma(i)=$ $f^{i}(x)$ for all $i \in \mathbb{Z}^{+}$. The extended $\sigma$ is called a full solution through $x$.

A set $S \subset X$ is invariant if $f(S)=S$. Given $N \subset X$, $\operatorname{Int}(N)$ denotes the interior of the set $N$ in $X$ and $\operatorname{cl}(N)$ the closure of the set $N$ in $X$. For $N \subset X$ its maximal invariant set is given by

$$
\operatorname{Inv}(N, f):=\left\{x \in N \mid \exists\left\{x_{n}\right\}_{n=-\infty}^{\infty} \subset N \text { such that } x_{n+1}=f\left(x_{n}\right) \text { and } x_{0}=x\right\} .
$$

A compact set $N \subset X$ is an isolating neighborhood, if $\operatorname{Inv}(N, f) \subset \operatorname{Int}(N)$. An invariant set $S$ is isolated, if there exists an isolating neighborhood $N$ such that $S=\operatorname{Inv}(N, f)$. 
Let $Y$ be a subset of $X$. The omega limit set of $Y$ is

$$
\omega(Y):=\bigcap_{k \in \mathbb{Z}^{+}} \operatorname{cl}\left(\left\{f^{k}(x) \mid x \in Y\right\}\right) .
$$

Given $x \in X$ and $\sigma$, a full left solution through $x$, the $\omega^{*}$-limit set for $\sigma$ is

$$
\omega^{*}(\sigma):=\bigcap_{k \in \mathbb{Z}^{+}} \operatorname{cl}(\sigma(-\infty,-k]) .
$$

A subset $A \subset S$ is an attractor in $S$, if there exists a neighborhood $U$ of $A$ such that

$$
\omega(U \cap S)=A \text {. }
$$

The dual repeller of $A$ in $S$ is

$$
A^{*}:=\{x \in S \mid \omega(x) \cap A=\emptyset\} .
$$

The pair $\left(A, A^{*}\right)$ is called an attractor-repeller pair in $S$. Finally, the chain recurrent set of $S$ is defined by

$$
\mathcal{R}(S):=\bigcap\left\{A \cup A^{*} \mid A \text { is an attractor in } S\right\} .
$$

In [1], the family of continuous maps $F^{\varepsilon}: \mathbb{R}^{n} \rightarrow \mathbb{R}^{n}$ given by

$$
F^{\varepsilon}(x)=f_{0}(x)+\sum_{i=1}^{m} \varepsilon^{i} f_{i}(x)+o\left(\varepsilon^{m+1}\right),
$$

where $f_{i}: \mathbb{R}^{n} \rightarrow \mathbb{R}^{n}, i=0, \ldots, m$ are continuous maps and $\varepsilon \in[0, \infty)$, is considered. Notice that $F^{0}=f_{0}$.

A compact set $N \subset \mathbb{R}^{n}$ is called a singular isolating neighborhood for $f_{0}$, if $N$ is not an isolating neighborhood for $f_{0}$, but there exists an $\bar{\varepsilon}>0$ such that, for all $\varepsilon \in(0, \bar{\varepsilon}], N$ is an isolating neighborhood for $F^{\varepsilon}$.

Let $N$ be a compact set. A point $x \in \operatorname{Inv}\left(N, f_{0}\right)$ is a slow exit point, if there exist a neighborhood $U \subset \mathbb{R}^{n}$ of $x, \bar{\varepsilon}>0$, and a positive integer $I:=I(\bar{\varepsilon}, U)$, such that for all $\varepsilon \in(0, \bar{\varepsilon}]$ and for all $y \in U$

$$
\left(F^{\varepsilon}\right)^{I}(y) \notin N .
$$

Similarly, the point $x \in \operatorname{Inv}\left(N, f_{0}\right)$ is a slow entrance point, if there exist neighborhood $U \subset \mathbb{R}^{n}$ of $x, \bar{\varepsilon}>0$, and a positive integer $I:=I(\bar{\varepsilon}, U)$, such that for every $\varepsilon \in(0, \bar{\varepsilon}]$, for all $y \in U$ and $z \in X$ such that

$$
\left(F^{\varepsilon}\right)^{I}(z)=y
$$

$z \notin N$

The next result which was proved in [1] shows how slow exit and slow entrance points are related to singular isolating neighborhoods.

Proposition 1. Let $F^{\varepsilon}: \mathbb{R}^{n} \rightarrow \mathbb{R}^{n}$ be a continuous family of continuous maps given by $(\star)$. Let $N$ be a compact set. If $\operatorname{Inv}\left(N, f_{0}\right) \cap \partial N$ consists of slow exit and entrance points, then $N$ is a singular isolating neighborhood.

In [2], Conley described conditions that imply the existence of slow exit and entrance points for the case of a family of continuous flows. For the discrete case, similar conditions can be determined. They were described in [1] and we will recall them now. First, 
we present the basic notation. Let $k$ and $m$ be positive integers. If $\alpha=\left(\alpha_{1}, \ldots, \alpha_{k}\right) \in$ $\{1, \ldots, m\}^{k}$, we define

$$
|\alpha|:=\sum_{j=1}^{k} \alpha_{j} .
$$

Given $i=1, \ldots, k$, the following set is considered:

$$
\mathcal{A}_{k, i}:=\left\{\alpha \in\{1, \ldots, m\}^{k}|| \alpha \mid \leq i\right\} .
$$

If $u_{i} \in \mathbb{R}^{n}, i=1, \ldots, n$, and $\alpha=\left(\alpha_{1}, \ldots, \alpha_{k}\right) \in\{1, \ldots, n\}^{k}$, the notation $u_{\alpha}$ will represent the vector $\left(u_{\alpha_{1}}, \ldots, u_{\alpha_{k}}\right) \in\left(\mathbb{R}^{n}\right)^{k}$.

Finally, we introduce the concept of averages of functions on invariant sets. Let $f$ : $\mathbb{R}^{n} \rightarrow \mathbb{R}^{n}$ be a continuous map, $S$ be a compact invariant set for $f$ and let $g: \mathbb{R}^{n} \rightarrow \mathbb{R}$ be a continuous function. The forward average of $g$ on $S$ is defined as

$$
\operatorname{FAve}(g, S):=\bigcap_{n \in \mathbb{Z}^{+}} \operatorname{cl}\left(\bigcup_{k \geq n}\left\{\frac{1}{k} \sum_{i=1}^{k} g\left(f^{i}(x)\right) \mid x \in S\right\}\right) .
$$

If FAve $(g, S) \subset(0, \infty)$, then we say that $g$ has strictly positive forward average on $S$. Similarly, we define the backward average of $g$ on $S$ by

$$
\operatorname{BAve}(g, S):=\bigcap_{n \in \mathbb{Z}^{+}} \operatorname{cl}\left(\bigcup_{k \geq n}\left\{\frac{1}{k} \sum_{i=1}^{k} g\left(f^{i}(y)\right) \mid f^{i}(y) \in S, i=1, \ldots, k \text {, and } f^{k}(y)=x\right\}\right) .
$$

As before, if $\operatorname{BAve}(g, S) \subset(0, \infty)$, then we say that $g$ has strictly positive backward average on $S$.

With this setup, we present the version of the definition of $C$-slow exit and $C$-slow entrance points for discrete dynamical systems.

Definition 1. Let $F^{\varepsilon}: \mathbb{R}^{n} \rightarrow \mathbb{R}^{n}$ be a continuous family of continuous maps given by $(\star)$ and $S=\operatorname{Inv}\left(N, f_{0}\right)$. A point $x \in S$ is a $C$-slow exit point (resp. $C$-slow entrance point) if there exist a compact set $K_{x} \subset S$, a neighborhood $U_{x}$ of $\mathcal{R}\left(K_{x}\right)$ and a $C^{m}$ function $\ell: \operatorname{cl}\left(U_{x}\right) \rightarrow \mathbb{R}$ such that

1. $\operatorname{Inv}\left(U_{x}, f_{0}\right)=\mathcal{R}\left(K_{x}\right)$.

2. $K_{x}$ is invariant under the map $f_{0}$ and $\omega\left(x, f_{0}\right) \subset K_{x}$ (resp. $\omega^{*}\left(\sigma, f_{0}\right) \subset K_{x}$, where $\sigma$ is a full solution through $x$ ).

3. $\ell\left(f_{0}(x)\right)=\ell(x)$.

4. If $L_{0}=\{z \mid \ell(z)=0\}$, then

$$
K_{x} \cap \operatorname{cl}\left(U_{x}\right)=S \cap L_{0} \cap \operatorname{cl}\left(U_{x}\right)
$$

and furthermore, $\left.\ell\right|_{K_{x} \cap \mathrm{cl}\left(U_{x}\right)}=0$, and $\left.\ell\right|_{S \cap \mathrm{cl}\left(U_{x}\right)} \leq 0$ (resp. $\geq 0$ ).

5. Let $g_{i}: \mathbb{R}^{n} \rightarrow \mathbb{R}$ be the continuous functions given by

$$
g_{i}(x)=\sum_{j=1}^{m} \sum_{\alpha \in \mathcal{A}_{j, i}} \frac{\ell^{(j)}\left(f_{0}(x)\right)}{j !} \cdot f_{\alpha}(x) .
$$

Then, $g_{j}=0$ if $j<m$, and $g_{m}$ has strictly positive forward (resp. backward) average on $\mathcal{R}\left(K_{x}\right)$. 
The main result of [1] takes the following form:

THEOREM 1. Let $F^{\varepsilon}: \mathbb{R}^{n} \rightarrow \mathbb{R}^{n}$ be a continuous family of continuous maps given by (*). Let $S=\operatorname{Inv}\left(N, f_{0}\right)$. Then

1. every $C$-slow exit point is a slow exit point.

2. Every $C$-slow entrance point is a slow entrance point.

3. A singular isolating neighborhood for the problem. Recall that our problem is to study the map $F^{\varepsilon}:[0,1] \times[0,3.3] \rightarrow \mathbb{R}^{2}$ given by

$$
F^{\varepsilon}(x, y)=\left(f(x, y), g^{\varepsilon}(x, y)\right)
$$

where

$$
\begin{aligned}
f(x, y) & =x(3.3-y)(1-x) \\
g^{\varepsilon}(x, y) & =y+\varepsilon y h(x, y)
\end{aligned}
$$

and $\varepsilon \geq 0$. Some additional requirements are made on the function $h$. Namely:

(1) there exists a unique solution of the system: $\left\{\begin{aligned}(3.3-y)(1-x) & =1 \\ h(x, y) & =0,\end{aligned}\right.$

(2) $\frac{\partial h}{\partial x}(x, y)>0$ and $\frac{\partial h}{\partial y}(x, y) \leq 0$, for all $(x, y) \in[0,1] \times[0,3.3]$,

(3) there exists $x^{*} \in(0,23 / 33)$ such that $h\left(x^{*}, 0\right)=0$.

It is not a coincidence that such conditions agree with the ones presented by Hutson and Mischaikow in [4]. They are the basic setup to construct a singular isolating neighborhood. They imply that we can control what will happen in the boundary of a compact set when the isolation property for $\varepsilon=0$ does not happen. Besides, our example fits in the framework of the work [4] but the reader should notice that we are able to prove that the attracting region is away from the axes for a much more general situation than the one described in [4] (cf. [4, Theorem 5.1]) since there are no additional requirements for the family of continuous maps we are working with.

We shall proceed in our analysis. Making $\varepsilon=0$, the dynamics of the map $f_{0}(x, y)=$ $(f(x, y), y)$ is dictated by the map $f$. Actually, for $\varepsilon=0$, the variable $y$ works as a parameter for $f$. For each $y$, the fixed point of $f_{y}(x):=f(x, y)$ is given by

$$
\bar{x}_{y}=1-\frac{1}{3.3-y} .
$$

Moreover, the stability of these fixed points is well known. Recall that $\bar{x}_{y}$ is a stable fixed point if $0.3<y \leq 3.3$ and $\bar{x}_{y}$ is unstable for $0 \leq y<0.3$. For $y \in[0,0.3), f_{y}$ also exhibits 2-periodic points, $x_{y}^{p}$ and $f_{y}\left(x_{y}^{p}\right)$, given by

$$
x_{y}^{p}=\frac{4.3-y+\sqrt{(4.3-y)(0.3-y)}}{2(3.3-y)}
$$

and

$$
f_{y}\left(x_{y}^{p}\right)=\frac{4.3-y-\sqrt{(4.3-y)(0.3-y)}}{2(3.3-y)} .
$$

In Figure 1, the representation of $\bar{x}_{y}, x_{y}^{p}$ and $f_{y}\left(x_{y}^{p}\right)$ is given. 


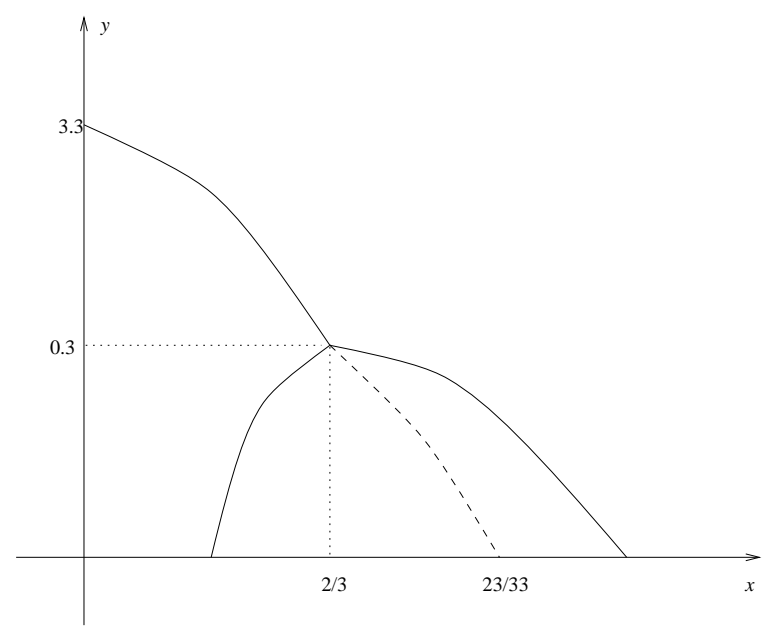

Fig. 1. Fixed point and 2-periodic point of $f_{y}$

Considering $\varepsilon>0$, the condition (1) imposed on the function $h$ implies that the fixed point of $F^{\varepsilon}$, for all $\varepsilon>0$, is $(\bar{x}, \bar{y})$ such that

$$
\left\{\begin{aligned}
h(\bar{x}, \bar{y}) & =0 \\
\bar{y} & =3.3-\frac{1}{1-\bar{x}} .
\end{aligned}\right.
$$

It is easy to construct a compact set $N$ that is not an isolating neighborhood for $\varepsilon=0$ and it is an isolating neighborhood for $\varepsilon>0$. To do that first observe that the conditions on the function $h$ imply that the curve $h(x, y)=0$ is $C^{1}$ with non-negative slope passing through the point $\left(x^{*}, 0\right)$ and $\lim _{(x, y) \rightarrow+\infty} h(x, y)=+\infty$. The curve $h(x, y)=0$ splits the plane into two regions. The region on the left of $h(x, y)=0$ is given by the points $(x, y)$ such that $h(x, y)<0$ and on the right, by $h(x, y)>0$ (see Hutson and Mischaikow [4]).
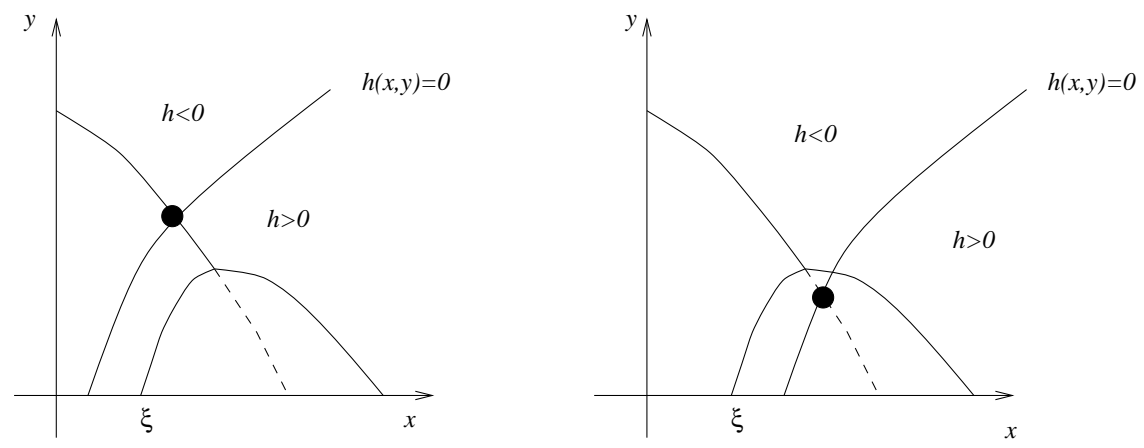

Fig. 2. The different possibilities for the curve $h(x, y)=0$

In Figure 2 the two possibilities for the position of the curve $h(x, y)=0$ with respect to the collection of points described in Figure 1 are presented. The points pictured with a black dot represent the fixed point $(\bar{x}, \bar{y})$ of $F^{\varepsilon}$ and $\xi=(43-\sqrt{129}) / 66$. 
Let $x_{0}, x_{1}, y_{0}$ and $y_{1}$ be positive real numbers such that

1. $x_{0}<x_{1}, y_{0}<y_{1}, x_{0}<\bar{x}$,

2. $\max \{0.3, \bar{y}\}<y_{1}<3.3-\frac{1}{1-x_{0}}, 0<y_{0}<\min \{0.3, \bar{y}\}$,

3. $h\left(1-\frac{1}{3.3-y_{0}}, y_{0}\right) \neq 0$ and $\left|h\left(f_{y}\left(x_{y_{0}}^{p}\right), y_{0}\right)\right| \neq\left|h\left(x_{y_{0}}^{p}, y_{0}\right)\right|$.

We will consider a compact set $N$ defined by $N:=\left[x_{0}, x_{1}\right] \times\left[y_{0}, y_{1}\right]$ (see Figure 3 ). The compact set $N$ is not an isolating neighborhood for $f_{0}$. The points $A_{1}, B, A_{2}$ and $D$ shown in Figure 3 prevent $N$ from being an isolating neighborhood. In fact, $B$ and $D$ are fixed points of $f_{0}$ and $A_{1}$ and $A_{2}$ are fixed points of $\left(f_{0}\right)^{2}$. Their coordinates can be easily computed and are:

$$
\begin{aligned}
A_{1} & =\left(\frac{4.3-y_{0}-\sqrt{\left(4.3-y_{0}\right)\left(0.3-y_{0}\right)}}{2\left(3.3-y_{0}\right)}, y_{0}\right) \\
B & =\left(\frac{2.3-y_{0}}{3.3-y_{0}}, y_{0}\right) \\
A_{2} & =\left(\frac{4.3-y_{0}+\sqrt{\left(4.3-y_{0}\right)\left(0.3-y_{0}\right)}}{2\left(3.3-y_{0}\right)}, y_{0}\right) \\
D & =\left(\frac{2.3-y_{1}}{3.3-y_{1}}, y_{1}\right)
\end{aligned}
$$

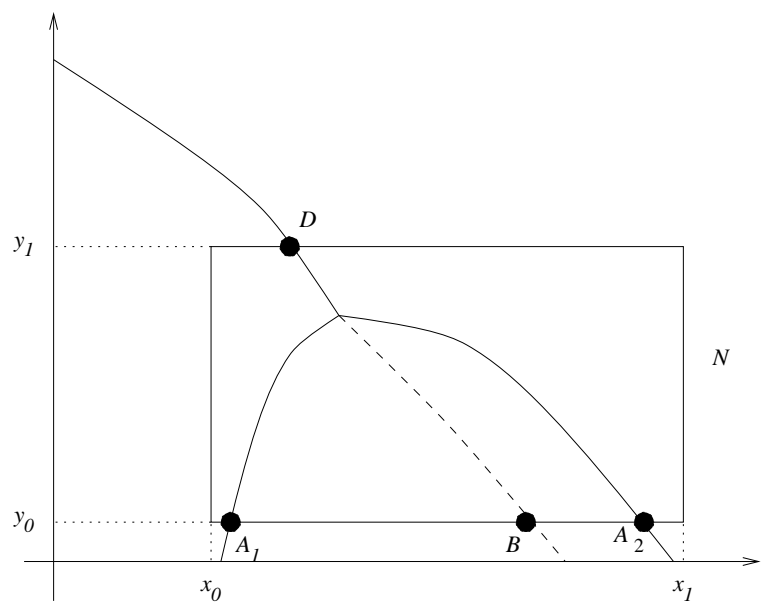

Fig. 3. A singular isolating neighborhood

Our goal is to show that $N$ is a singular isolating neighborhood. Since $\operatorname{Inv}\left(N, f_{0}\right) \cap$ $\partial N=\left\{A_{1}, B, A_{2}, D\right\}$, we need to prove that there exists $\bar{\varepsilon}>0$ such that if $\varepsilon \in(0, \bar{\varepsilon}]$, then $A_{1}, B, A_{2}, D \notin \operatorname{Inv}\left(N, F^{\varepsilon}\right)$. To do this, Theorem 1 will be used. In each case, the main step is to construct a function $\ell$. In the notation of Theorem 1 ,

$$
\begin{aligned}
& f_{0}(x, y)=(x(3.3-y)(1-x), y) \\
& f_{1}(x, y)=(0, y h(x, y))
\end{aligned}
$$


The next set of propositions shows the analysis for each of these points.

Proposition 2. The point $D$ is a $C$-slow exit point.

Proof. Consider $K_{D}=\{D\}$. Notice that $\mathcal{R}\left(K_{D}\right)=K_{D}$. Let $U_{D}$ be a small neighborhood of the point $D$. The definition of the function $\ell: \operatorname{cl}\left(U_{D}\right) \rightarrow \mathbb{R}$ is $\ell(x, y)=-y+y_{1}$, where $y_{1}$ is the second coordinate of the point $D$. One can easily verify the conditions 1 , 2, 3 and 4 of Definition 1. It remains to prove the last condition. Notice that

$$
\nabla \ell(x, y)=\left(\begin{array}{r}
0 \\
-1
\end{array}\right)
$$

Therefore

$$
g_{1}(x, y)=\nabla \ell\left(F^{\varepsilon}(x, y)\right) \cdot f_{1}(x, y)=-y h(x, y)
$$

which implies

$$
\operatorname{FAve}\left(g_{1}, \mathcal{R}\left(K_{D}\right)\right)=-y_{1} h\left(\frac{2.3-y_{1}}{3.3-y_{1}}, y_{1}\right)
$$

Since the point $\left(\frac{2.3-y_{1}}{3.3-y_{1}}, y_{1}\right)$ was chosen on the left side of the curve $h(x, y)=0$, we have $\operatorname{FAve}\left(g_{1}, \mathcal{R}\left(K_{D}\right)\right)>0$.

Proposition 3. The point $B$ is a $C$-slow entrance point.

Proof. This case is similar to the previous one. The function $\ell$ considered is $\ell(x, y)=$ $y-y_{0}$.

Proposition 4. If $x^{*} \in(0,(43-\sqrt{129}) / 66)$, then $A_{1}$ and $A_{2}$ are $C$-slow entrance points. If $x^{*} \in((43-\sqrt{129}) / 66,23 / 33)$, then there are two possibilities:

1. if $-h\left(x_{A_{1}}, y_{0}\right)<h\left(x_{A_{2}}, y_{0}\right)$, then $A_{1}$ and $A_{2}$ are $C$-slow exit points.

2. if $-h\left(x_{A_{1}}, y_{0}\right)>h\left(x_{A_{2}}, y_{0}\right)$, then $A_{1}$ and $A_{2}$ are $C$-slow entrance points.

Proof. Consider the case $x^{*} \in(0,(43-\sqrt{129}) / 66)$ and let $x=A_{i}, i=1,2$. In both cases, take $S=\left\{A_{1}, A_{2}\right\}$. Also notice that $\mathcal{R}\left(K_{x}\right)=K_{x}$. Let $U_{x}$ be a small neighborhood of the point $x$. The function $\ell$ is defined as $\ell(x, y)=y-y_{0}$. The conditions $1,2,3$ and 4 of Definition 1 for $C$-slow entrance points are satisfied. To finish, the condition 5 needs to be verified. We have

$$
\nabla \ell(x, y)=\left(\begin{array}{l}
0 \\
1
\end{array}\right)
$$

and

$$
g_{1}(x, y)=\nabla \ell\left(F^{\varepsilon}(x, y)\right) \cdot f_{1}(x, y)
$$

Therefore,

$$
\operatorname{BAve}\left(g_{1}, \mathcal{R}\left(K_{x}\right)\right)=\frac{1}{2} y_{0}\left[h\left(x_{A_{1}}, y_{0}\right)+h\left(x_{A_{2}}, y_{0}\right)\right]
$$

where $\left(x_{A_{1}}, y_{0}\right)$ and $\left(x_{A_{2}}, y_{0}\right)$ are the coordinates of $A_{1}$ and $A_{2}$, respectively. Since $A_{1}$ and $A_{2}$ lie on the right of the curve $h(x, y)=0, h\left(x_{A_{1}}, y_{0}\right)>0$ and $h\left(x_{A_{2}}, y_{0}\right)>0$. 
For the case $x^{*} \in((43-\sqrt{129}) / 66,23 / 33)$ the choice of the function $\ell$ will depend on the sign of $h\left(x_{A_{1}}, y_{0}\right)+h\left(x_{A_{1}}, y_{0}\right)$. Let

$$
\ell(x, y)=\left\{\begin{array}{rrr}
y-y_{0} & \text { if } & -h\left(x_{A_{1}}, y_{0}\right)<h\left(x_{A_{1}}, y_{0}\right) \\
-y+y_{0} & \text { if } & -h\left(x_{A_{1}}, y_{0}\right)>h\left(x_{A_{1}}, y_{0}\right)
\end{array}\right.
$$

The rest of the proof follows the steps done before.

Combining Theorem 1, Propositions 1, 2, 3 and 4, we conclude

COROLlary 1 . The compact set $N=\left[x_{0}, x_{1}\right] \times\left[y_{0}, y_{1}\right]$, where $x_{0}, x_{1}, y_{0}$ and $y_{1}$ as above, is a singular isolating neighborhood for $f_{0}$.

4. An index computation for a family of functions $h_{\lambda}$. In this section we will consider the family of continuous functions $h_{\lambda}: \mathbb{R} \rightarrow \mathbb{R}$ given by

$$
h_{\lambda}(x, y):=x^{n}-\lambda^{n},
$$

where $\lambda \in[0.4,0.7]$ and $n$ is a fixed positive integer. Notice that the map $F^{\varepsilon}$ has an extra parameter. Therefore the notation $F_{\lambda}^{\varepsilon}$ is used. For each $\lambda \in[0.4,23 / 33]$, the only fixed point of $F_{\lambda}^{\varepsilon}$ is given by

$$
\begin{aligned}
& \bar{x}_{\lambda}=\lambda \\
& \bar{y}_{\lambda}=3.3-\frac{1}{1-\lambda} .
\end{aligned}
$$

REMARK 1. For $\lambda \in(23 / 33,0.7]$ the second coordinate of the fixed point is negative.

The construction of the compact set $N$ done in the previous section can be repeated and we obtain a compact set that is not an isolating neighborhood for $\varepsilon=0$ and it is an isolating neighborhood for $\varepsilon>0$. Even though the continuous map we are studying has an extra parameter $\lambda$, we can add some requirements that imply that $N$ does not depend on $\lambda$. We assume that the points $x_{0}, x_{1}, y_{0}$ and $y_{1}$ are positive real numbers such that

1. $x_{0}<x_{1}, y_{0}<y_{1}$,

2. $3.3-\frac{1}{1-0.4}<y_{1}<3.3-\frac{1}{1-x_{0}}$, and

3. $y_{0}>0.3$.

Then define $N:=\left[x_{0}, x_{1}\right] \times\left[y_{0}, y_{1}\right]$ as before. The picture in Figure 3 is also a geometric representation of $N$. In what follows $\lambda_{0}:=1-\frac{1}{1-y_{0}}$. Notice that $\lambda_{0}<23 / 33$. Propositions 2, 3 and 4 imply

Corollary 2. 1. For all $\lambda \in(0.4,0.7)$ the point $D$ is a $C$-slow exit point.

2. If $\lambda \in\left(0.4, \lambda_{0}\right)$, then $B$ is a $C$-slow entrance point. If $\lambda \in\left(\lambda_{0}, 0.7\right)$, then $B$ is a $C$-slow exit point.

3. If $\lambda \in\left(0.4, \lambda_{0}\right)$, then $A_{1}$ and $A_{2}$ are $C$-slow entrance points. If $\lambda \in\left(\lambda_{0}, 0.7\right)$, then $A_{1}$ and $A_{2}$ are $C$-slow exit points.

REMARK 2. The same type of arguments can be used to prove that other compact sets are singular isolating neighborhoods. Below, a new compact set will be considered and following the steps done in Section 3 we conclude that it is also a singular isolating neighborhood. 
Now, we start to study the fixed points $\left(\bar{x}_{\lambda}, \bar{y}_{\lambda}\right)$, for $\lambda \in(0.4,23 / 33)$. The Jacobian matrix of $F_{\lambda}^{\varepsilon}(x, y)$, denoted by $D F_{\lambda}^{\varepsilon}(x, y)$, is

$$
D F_{\lambda}^{\varepsilon}(x, y)=\left[\begin{array}{cc}
(3.3-y)(1-x)-x(3.3-y) & -x(1-x) \\
\varepsilon n y x^{n-1} & 1+\varepsilon\left(x^{n}-\lambda^{n}\right)
\end{array}\right] .
$$

Computing it at the fixed point $\left(\bar{x}_{\lambda}, \bar{y}_{\lambda}\right)$ we obtain

$$
D F_{\lambda}^{\varepsilon}\left(\bar{x}_{\lambda}, \bar{y}_{\lambda}\right)=\left[\begin{array}{cc}
1-\lambda\left(3-\bar{y}_{\lambda}\right) & -\lambda(1-\lambda) \\
\varepsilon n y \lambda^{n-1} & 1
\end{array}\right] .
$$

The eigenvalues of $D F_{\lambda}^{\varepsilon}\left(\bar{x}_{\lambda}, \bar{y}_{\lambda}\right)$ are given by

$$
x_{\varepsilon, \lambda}^{+}=\frac{2-3 \lambda+\sqrt{\lambda^{2}-\varepsilon 4 n \lambda^{n}(1-\lambda)^{2}(2.3-3.3 \lambda)}}{2(1-\lambda)}
$$

and

In what follows the constant $c$ denotes

$$
x_{\varepsilon, \lambda}^{-}=\frac{2-3 \lambda-\sqrt{\lambda^{2}-\varepsilon 4 n \lambda^{n}(1-\lambda)^{2}(2.3-3.3 \lambda)}}{2(1-\lambda)} .
$$

$$
c:=\sqrt{1-\varepsilon 4 n \lambda^{n-2}(1-\lambda)^{2}(2.3-3.3 \lambda)}
$$

Notice that $0<c<1$.

In the next three lemmas the behavior of $x_{\varepsilon, \lambda}^{+}$and $x_{\varepsilon, \lambda}^{-}$as functions of the parameters $\lambda$ and $\varepsilon$ is studied.

Lemma 1. 1. If $\lambda \in(0.4,2 / 3)$, then $0<x_{\varepsilon, \lambda}^{+}<1$.

2. If $\lambda \in(2 / 3,23 / 33)$, then there exists $\bar{\varepsilon}_{0}>0$ which does not depend on $\lambda$ such that $0<x_{\varepsilon, \lambda}^{+}<1$ for all $\varepsilon \in\left(0, \bar{\varepsilon}_{0}\right)$.

Proof. Notice that

$$
2-3 \lambda+\lambda c<2(1-\lambda) \quad \text { if and only if } \quad c<1 .
$$

Therefore, for all $\lambda \in(0.4,23 / 33)$, we have $x_{\varepsilon, \lambda}^{+}<1$. In the same way

$$
0<x_{\varepsilon, \lambda}^{+} \quad \text { if and only if } 2-3 \lambda+\lambda c>2(1-\lambda)>0 .
$$

If $\lambda \in(0.4,2 / 3)$, then $2-3 \lambda<0$ and we have proved the first part of the lemma.

To finish, the case $\lambda \in(2 / 3,23 / 33)$ is considered. Let $\bar{\varepsilon}_{0}>0$ be such that

$$
\bar{\varepsilon}_{0}<\frac{210}{n} \frac{33^{n-2}}{23^{n}}
$$

and consider $\varepsilon \in\left(0, \bar{\varepsilon}_{0}\right]$. Since $2-3 \lambda>0$, we only need to verify that $\lambda^{2} c^{2}>(2-3 \lambda)^{2}$ and this is equivalent to proving that

$$
-2 \lambda^{2}+3 \lambda-1-\varepsilon n \lambda^{n}(1-\lambda)^{2}(2.3-3.3 \lambda)>0 .
$$

A simple computation shows that

This concludes the proof.

$$
\begin{aligned}
-2 \lambda^{2}+3 \lambda-1-\varepsilon n \lambda^{n}(1-\lambda)^{2}(2.3-3.3 \lambda) & >\frac{7}{99}-\frac{\varepsilon n}{30} \frac{23^{n}}{33^{n}} \\
& >\frac{7}{99}-\frac{\bar{\varepsilon}_{0} n}{30} \frac{23^{n}}{33^{n}}>0 .
\end{aligned}
$$


Lemma 2. If $\lambda \in(0.4,2 / 3)$, then $\left|x_{\varepsilon, \lambda}^{-}\right|<1$ for all $\varepsilon>0$.

Proof. Since $c>0$ and $\lambda \in(0.4,2 / 3)$ implies that $c<4 / \lambda-5$, we have $\left|x_{\varepsilon, \lambda}^{-}\right|<1$ for all $\varepsilon>0$.

In what follows, $\mu_{0}$ denotes a real number in the interval $(1 / 48,1 / 33)$.

Lemma 3. If $\lambda \in\left(2 / 3+\mu_{0}, 23 / 33\right)$, then there exists $\bar{\varepsilon}_{1}>0$ which does not depend on $\lambda$ such that $x_{\varepsilon, \lambda}^{-}<-1$ for all $\varepsilon \in\left(0, \bar{\varepsilon}_{1}\right)$.

Proof. Let $\bar{\varepsilon}_{1}>0$ be such that

$$
\bar{\varepsilon}_{1}<\frac{180}{n} \frac{33^{n-1}}{23^{n}} \frac{\left(48 \mu_{0}-1\right)}{\left(1-\mu_{0}\right)^{2}\left(1-33 \mu_{0}\right)}
$$

and consider $\varepsilon \in\left(0, \bar{\varepsilon}_{1}\right]$.

We need to verify that for all $\lambda \in\left(2 / 3+\mu_{0}, 23 / 33\right), 4-5 \lambda<c \lambda$. Since $4-5 \lambda>0$,

$$
\begin{aligned}
4-5 \lambda<c \lambda & \Longleftrightarrow(4-5 \lambda)^{2}<c^{2} \lambda^{2} \\
& \Longleftrightarrow 3 \lambda^{2}-5 \lambda+2+\frac{\varepsilon n}{2} \lambda^{n}(1-\lambda)^{2}(2.3-3.3 \lambda)<0 .
\end{aligned}
$$

Again algebraic computation shows that

$$
\begin{aligned}
3 \lambda^{2}-5 \lambda & +2+\frac{\varepsilon n}{2} \lambda^{n}(1-\lambda)^{2}(2.3-3.3 \lambda) \\
& <\frac{2}{33}-\frac{32 \mu_{0}}{11}+\frac{\varepsilon n}{180} \frac{23^{n}}{33^{n}}\left(1-\mu_{0}\right)^{2}\left(1-33 \mu_{0}\right)<0 .
\end{aligned}
$$

This concludes the proof.

Now, we are ready for the computation of the cohomological Conley index for the fixed point $\left(\bar{x}_{\lambda}, \bar{y}_{\lambda}\right)$. We refer to [6] and [8] for definitions and basic results of the Conley index theory for discrete dynamical systems.

Proposition 5. Let $\lambda$ and $\bar{\mu}_{0}$ be real numbers such that $0.4<\lambda<\lambda_{0}$ and $1 / 48<$ $\bar{\mu}_{0}<\min \left\{-\lambda_{0}+2 / 3,1 / 33\right\}$. Let $S_{\lambda}^{\varepsilon}=\left\{\left(\bar{x}_{\lambda}, \bar{y}_{\lambda}\right)\right\}$. There exists $\bar{\varepsilon}>0$ such that if $\varepsilon \in(0, \bar{\varepsilon}]$, then $S_{\lambda}^{\varepsilon}$ is an isolated invariant set and

$$
\operatorname{Con}\left(S_{\lambda}^{\varepsilon}, F_{\lambda}^{\varepsilon}\right)=\left\{\begin{array}{lll}
(0,0) & \text { if } \quad \lambda \in(0.4,2 / 3), \\
(\mathbb{Z},- \text { Id }) & \text { if } \quad \lambda \in\left(2 / 3+\bar{\mu}_{0}, \lambda_{0}\right) .
\end{array}\right.
$$

Proof. It is trivial that there exists $\bar{\varepsilon}>0$ such that for $\varepsilon \in(0, \bar{\varepsilon}], S_{\lambda}^{\varepsilon}$ is an isolated invariant set for $F_{\lambda}^{\varepsilon}$. The computation of the Conley index of $S_{\lambda}^{\varepsilon}$ follows from Lemmas 1, 2,3 and [6, Theorem 3.1].

We will use the continuation property of the Conley index (see [6] and [8]) to prove our last result.

Corollary 3. Let $N$ be the singular isolating neighborhood defined in the Section 3. If $\lambda \in\left(2 / 3+\bar{\mu}_{0}, \lambda_{0}\right)$, then

$$
S_{\lambda}^{\varepsilon} \neq \operatorname{Inv}\left(N, F_{\lambda}^{\varepsilon}\right) .
$$

Proof. In section 3 we proved that there exists $\bar{\varepsilon}>0$ such that if $\varepsilon \in(0, \bar{\varepsilon}]$, then $N$ is an isolating neighborhood for $F_{\lambda}^{\varepsilon}$ for all $\lambda \in\left(0.4, \lambda_{0}\right)$. Therefore, fix $\varepsilon_{0} \in(0, \bar{\varepsilon}]$. The 
continuation property of the Conley index implies that

$$
\operatorname{Con}\left(N, F_{\lambda}^{\varepsilon_{0}}\right)=\operatorname{Con}\left(N, F_{\mu}^{\varepsilon_{0}}\right),
$$

for all $\lambda, \mu \in\left(0.4, \lambda_{0}\right)$. Proposition 5 implies the result.

Acknowledgments. I wish to thank Professors Vivian Hutson and Konstantin Mischaikow for many fruitful discussions during the Workshop on Conley Index held at Banach Center, Warsaw, in June 1997.

\section{References}

[1] M. Burke, M. C. Carbinatto and K. Mischaikow, Singular isolating neighborhoods for continuous maps, in preparation.

[2] C. Conley, A qualitative singular perturbation theorem, Global Theory of Dynamical Systems, eds. Z. Nitecki and C. Robinson, Lectures Notes in Math., 819, Springer-Verlag 1980, 65-89.

[3] C. Conley, Isolated Invariant Sets and the Morse Index, CBMS Reg. Conf. Ser. in Math., 38, AMS, Providence, 1978.

[4] V. Hutson and K. Mischaikow, An approach to practical persistence, J. Math. Biol. 37 (1998), 447-466.

[5] K. Mischaikow, M. Mrozek, J. Reineck, Singular index pairs, J. Dyn. Diff. Eq., to appear.

[6] M. MrozeK, Leray functor and cohomological Conley index for discrete dynamical systems, Trans. AMS 318 (1990), 149-178.

[7] M. Mrozek and K. P. Rybakowski, A cohomological Conley index for maps on metric spaces, J. Differential Equations 89 (1991), 143-171.

[8] A. Szymczak, The Conley index for discrete semidynamical systems, Topology Appl. 66 (1996), 215-240. 